\title{
Achievement of Target Serum Uric Acid Levels and Factors Associated with Therapeutic Failure among Japanese Men Treated for Hyperuricemia/Gout
}

\author{
Akiko Katayama $^{1}$, Hirohide Yokokawa ${ }^{1}$, Hiroshi Fukuda ${ }^{1}$, Yoshiki Ono ${ }^{2}$, Hiroshi Isonuma ${ }^{1}$, \\ Teruhiko Hisaoka ${ }^{1}$ and Toshio Naito ${ }^{1}$
}

\begin{abstract}
:
Objective To assess the rate of successfully achieving treatment goals among Japanese men with hyperuricemia/gout and identify factors influencing the success rate.

Methods This cross-sectional study, conducted from January to December 2012, examined the serum uric acid (SUA) levels and clinical characteristics of 2,103 men with hyperuricemia/gout selected from an initial population of 136,770 individuals who participated in a workplace health checkup. The success rates (defined as SUA $\leq 6.0 \mathrm{mg} / \mathrm{dL}$ ) were calculated, and a multivariate analysis was used to identify factors associated with "therapeutic failure" to achieve target SUA levels.

Results The rate of successfully achieving the target SUA level was $37.5 \%$. The body mass index (BMI) was significantly associated with therapeutic failure $[25.0 \leq$ Category $(\mathrm{C}) 2<27.5$, adjusted odds ratio $(\mathrm{AOR})=$ $1.35 ; 27.5 \leq \mathrm{C} 3<30.0, \mathrm{AOR}=1.69 ; \mathrm{C} 4 \geq 30.0, \mathrm{AOR}=1.94$; relative to $\mathrm{C} 1<25.0]$. A significant positive association was also observed between waist circumference (WC) and therapeutic failure $(85 \leq \mathrm{C} 2<90, \mathrm{OR}=1.29 ; 90 \leq$ $\mathrm{C} 3<95, \mathrm{OR}=1.41 ; 95 \leq \mathrm{C} 4, \mathrm{OR}=2.28$; relative to $\mathrm{C} 1<85.0 \mathrm{~cm}$ ). Those with higher $\mathrm{BMI} / \mathrm{WC}$ measurements were significantly more likely to have higher SUA levels than those with lower such measurements. The ongoing intake of dyslipidemia medication was identified as a protective factor against therapeutic failure.

Discussion Our findings suggest a possible association between obesity and therapeutic failure, underscoring the importance of maintaining lipid profiles as part of managing SUA levels. Better management of both obesity and dyslipidemia may prevent future cardiovascular disorders by ensuring healthier SUA levels.
\end{abstract}

Key words: uric acid, obesity, achievement rate, lifestyle-related disorder, prevention, epidemiology

(Intern Med 58: 1225-1231, 2019)

(DOI: 10.2169/internalmedicine.1899-18)

\section{Introduction}

Hyperuricemia is a major risk factor for cardiovascular disease (CVD), chronic kidney disease (CKD), gout, and metabolic syndrome (1-4), and some experimental studies have indicated a modulatory or causal role of hyperuricemia in these disorders (5-7). Primary hospitalization rates for gout have increased substantially for the past nearly two decades in the United States, while those for rheumatoid arthritis have declined (8). The prevalence of hyperuricemia is estimated to be approximately $25 \%$ among Japanese adult men, with the number of patients with gout in 2004 having increased by 2.1 -fold compared to 1995 and by 3.4-fold compared to 1986 (9). According to the guidelines of The Japanese Society of Gout and Nucleic Acid Metabolism, hyperuricemia is defined as a serum uric acid (SUA) level $\geq$ $7.0 \mathrm{mg} / \mathrm{dL}$. Based on several previous empirical studies (9-11), it is recommended that the SUA levels be kept at $\leq 6.0 \mathrm{mg} / \mathrm{dL}$ among patients with a high risk of gout in order to ensure primary or secondary prevention. In fact, several intervention studies have demonstrated that SUA-lowering

${ }^{1}$ Department of General Medicine, Juntendo University School of Medicine, Japan and ${ }^{2}$ Department of Health Screening, Tokyo Health Service Association, Japan

Received: July 30, 2018; Accepted: October 15, 2018; Advance Publication by J-STAGE: January 10, 2019

Correspondence to Dr. Hirohide Yokokawa, yokokawa@pa3.so-net.ne.jp 


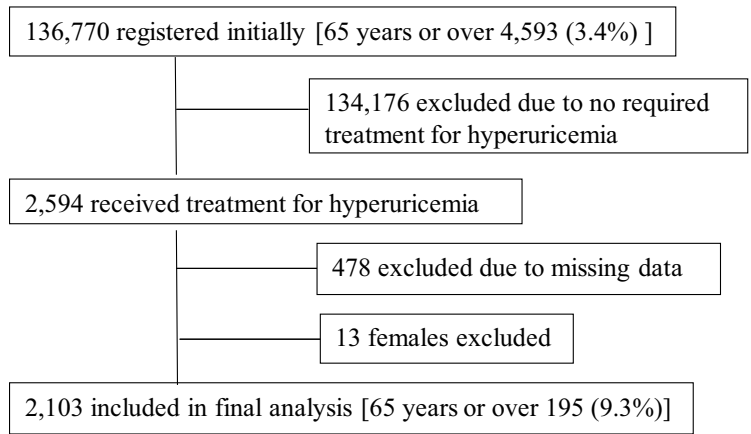

Figure 1. Patient registration and flowchart of the study.

agents reduce the incidence and recurrence of gout (12). Thus, these treatment guidelines suggest that the management of hyperuricemia/gout is an important way to help prevent these disorders. However, limited evidence is available on the actual status of SUA management in Japan.

The present study therefore aimed to assess the rate of successfully achieving treatment goals and explore factors associated with therapeutic failure among Japanese men with hyperuricemia/gout.

\section{Materials and Methods}

This was a cross-sectional study screening 136,770 Japanese adults [ $\geq 65$ years of age; $4,593(3.4 \%)]$ who participated in a workplace health checkup conducted by the Tokyo Health Service Association from January to December 2012 in Tokyo, Japan. Of the 2,594 subjects deemed to have hyperuricemia/gout, 478 were excluded due to missing data on SUA, and 13 women were additionally excluded because they comprised a proportion that was too small for an analysis. Ultimately, 2,103 men were included in the present study [ $\geq 65$ years of age; $195(9.3 \%)]$ (Fig. 1).

\section{Variables}

The height, weight, body mass index (BMI), and waist circumference (WC) were measured with participants in the standing position. The BMI was calculated by dividing the body weight $(\mathrm{kg})$ by the height squared $\left(\mathrm{m}^{2}\right)$. The mean systolic blood pressure (SBP) and diastolic blood pressure (DBP) were calculated from the means of two upper arm blood pressure measurements taken from participants who had been seated for at least five minutes. Serum levels of total cholesterol (mg/dL; TC), high-density lipoprotein cholesterol (mg/dL; HDL-C), low-density lipoprotein cholesterol $(\mathrm{mg} / \mathrm{dL} ; \mathrm{LDL}-\mathrm{C})$, and triglycerides $(\mathrm{mg} / \mathrm{dL} ; \mathrm{TG})$ were also measured. LDL-C was estimated using the Friedewald equation (TC - HDL-C - TG/5) (13). Hemoglobin A1c (HbA1c) levels were determined by high-performance liquid chromatography using an automated analyzer. HbA1c [Japanese Diabetes Society (JDS; \%)] values were converted to National Glycohemoglobin Standardization Program (NGSP) equivalent values using the following formula: HbA1c $(\mathrm{NGSP})(\%)=1.02 \times \mathrm{HbA} 1 \mathrm{c}(\mathrm{JDS})(\%)+0.25 \%$ (14). SUA was measured with the uricase-peroxidase method. The estimated glomerular filtration rate (eGFR) was calculated using the Japanese GFR equation: eGFR $\left(\mathrm{mL} / \mathrm{min} / 1.73 \mathrm{~m}^{2}\right)=194 \times$ $\mathrm{Cr}^{-1.094} \times$ age $\mathrm{e}^{-0.287}$.

Participants were asked to complete a self-administered questionnaire that addressed healthy lifestyle characteristics based on Breslow's seven health practices (15). These characteristics can be used to assess lifestyle health, and strong associations have been found between healthy lifestyle items and successful blood pressure control among patients with hypertension (16). Healthy lifestyle items in the questionnaire included non-daily alcohol consumption, non-smoker status, exercise frequency of two or more times per week, a BMI of $18.5-24.9 \mathrm{~kg} / \mathrm{m}^{2}$, adequate sleep duration, daily breakfast consumption, and no snacking between meals $(15,16)$.

From the self-administered questionnaire, we also collected information on a present medical history of comorbidities, such as hyperuricemia/goat, hypertension, diabetes mellitus, dyslipidemia, coronary artery disease, cerebrovascular disease, and kidney disease. If participants acknowledged having these comorbidities, we registered them as having a present medical history of these comorbidities.

\section{Statistical analyses}

The results are presented as mean \pm standard deviation (SD) for continuous variables or prevalence (\%) for categorical variables. The BMI $\left(\mathrm{kg} / \mathrm{m}^{2}\right)$ and $\mathrm{WC}(\mathrm{cm})$ were divided into the following categories: BMI $\left(\mathrm{kg} / \mathrm{m}^{2}\right)=\mathrm{C} 1<25.0$, $25.0 \leq \mathrm{C} 2<27.5,27.5 \leq \mathrm{C} 3<30.0,30.0 \leq \mathrm{C} 4$; WC $(\mathrm{cm})=\mathrm{C} 1<85$, $85 \leq \mathrm{C} 2<90,90 \leq \mathrm{C} 3<95,95 \leq \mathrm{C} 4$. The target SUA level was defined as $\leq 6.0 \mathrm{mg} / \mathrm{dL}(356.9 \mu \mathrm{mol} / \mathrm{L})$ based on the treatment guidelines. The "controlled group" and "uncontrolled group" comprised those with SUA levels $\leq 6.0$ and $>6.0 \mathrm{mg}$ / dL, respectively (9). Factors associated with therapeutic failure in achieving target SUA levels were identified using odds ratios (ORs) and 95\% confidence intervals (CIs) for each item, with a univariate logistic regression analysis and two multivariate models providing adjusted ORs (AORs) and 95\% CIs. Model 1 was adjusted for age (years), BMI, hypertension medication (yes), diabetic medication (yes), dyslipidemia medication (yes), alcohol consumption (nondaily drinker), smoking (non-smoker), and eGFR ( $\mathrm{mL} / \mathrm{min} /$ $1.73 \mathrm{~m}^{2}$ ). Model 2 was adjusted for age (years), WC, hypertension medication (yes), diabetic medication (yes), dyslipidemia medication (yes), alcohol consumption (non-daily drinker), smoking (non-smoker), and eGFR (mL/min/1.73 $\left.\mathrm{m}^{2}\right)$.

The SUA levels were compared between $\mathrm{C} 1$ and other quartiles with Dunnett's method. Trends in $p$ values were estimated using the Jonckheere-Terpstra test for continuous variables. For the two-tailed Student's $t$-test, $\mathrm{p}<0.05$ was considered statistically significant. All statistical analyses were performed using the Statistical Package for Social Sciences, version 22 (SPSS, Chicago, USA).

The research protocol was reviewed and approved by the 
Table 1. Participant Characteristics $(n=2,103)$.

\begin{tabular}{|c|c|c|c|}
\hline & \multicolumn{2}{|c|}{$\begin{array}{l}\text { Mean }(\mathrm{SD}) \text { or } \mathrm{N}(\%) \\
\text { Uric acid }(\mathrm{mg} / \mathrm{dL})\end{array}$} & \\
\hline & $\leq 6.0(\mathrm{n}=788)$ & $>6.0(\mathrm{n}=1,315)$ & \\
\hline Age (years) & $54.2(9.5)$ & $53.1(9.5)$ & $* *$ \\
\hline \multicolumn{4}{|l|}{ Anthropometric measurements } \\
\hline Body mass index $\left(\mathrm{kg} / \mathrm{m}^{2}\right)$ & $25.1(3.3)$ & $25.8(3.7)$ & $* *$ \\
\hline Waist circumference $(\mathrm{cm})$ & $88.5(8.4)$ & $90.3(9.4)$ & $* *$ \\
\hline \multicolumn{4}{|l|}{ Healthy lifestyle characteristics } \\
\hline Alcohol consumption (non-daily drinker) & $443(56.2)$ & $752(57.2)$ & \\
\hline Smoking behavior (non-smoker) & $548(76.9)$ & $920(77.2)$ & \\
\hline Exercise frequency (2 times or more per week) & $360(50.5)$ & $597(50.1)$ & \\
\hline Body mass index (18.5-24.9) & $428(54.3)$ & $598(45.5)$ & $* *$ \\
\hline Adequate sleep duration (yes) & $474(66.6)$ & $755(63.5)$ & \\
\hline Breakfast (every morning) & $602(84.6)$ & $1,001(84.1)$ & \\
\hline Snack between meals (no) & $649(91.0)$ & $1,074(90.2)$ & \\
\hline $\begin{array}{l}\text { Proportion of participants with } 6 \text { or } 7 \text { total } \\
\text { number of healthy lifestyle items }\end{array}$ & $208(29.3)$ & $300(25.3)$ & \\
\hline Hypertensive medication (yes) & $313(43.9)$ & $525(44.0)$ & \\
\hline Systolic blood pressure (mmHg) & $128.7(17.2)$ & $129.9(17.6)$ & \\
\hline Diastolic blood pressure (mmHg) & $80.6(10.7)$ & $81.8(11.2)$ & $*$ \\
\hline Diabetic medication (yes) & $49(6.9)$ & $79(6.6)$ & \\
\hline Hemoglobin A1c (\%) & $5.4(0.7)$ & $5.4(0.7)$ & \\
\hline Dyslipidemia medication (yes) & $223(31.3)$ & $322(27.0)$ & $*$ \\
\hline Total cholesterol (mg/dL) & $198.9(32.1)$ & $204.5(34.3)$ & $* *$ \\
\hline High density cholesterol (mg/dL) & $56.0(13.5)$ & $54.1(13.7)$ & $* *$ \\
\hline Low density cholesterol (mg/dL) & $114.2(29.9)$ & $117.8(29.6)$ & $* *$ \\
\hline Triglyceride (mg/dL) & $168.8(149.6)$ & $175.5(145.7)$ & \\
\hline Uric acid (mg/dL) & $5.2(0.7)$ & $7.2(0.9)$ & $* *$ \\
\hline \multicolumn{4}{|l|}{ Organ damage/cardiovascular disease } \\
\hline Heart & $38(4.8)$ & $60(4.6)$ & \\
\hline Brain & $11(1.4)$ & $14(1.1)$ & \\
\hline Kidney & $2(0.3)$ & $12(0.9)$ & \\
\hline eGFR (mL/min/1.73 m²) & $73.5(15.1)$ & $70.2(15.3)$ & $* *$ \\
\hline
\end{tabular}

Ethics Committee of the Tokyo Health Service Association, and written informed consent was obtained from all participants.

\section{Results}

The proportion of participants in the controlled group [SUA level $\leq 6.0 \mathrm{mg} / \mathrm{dL}(356.9 \mu \mathrm{mol} / \mathrm{L})$ ] was $37.5 \%$, which represented the rate of successfully achieving the treatment goal. The mean ages (SD) of the controlled and uncontrolled groups were 54.2 (9.5) and 53.1 (9.5) years, respectively (Table 1). The uncontrolled group had significantly higher mean values for the BMI, WC, DPB, TC, and LDL-C and significantly lower values for the HDL-C and eGFR than the controlled group. The proportion of those taking dyslipidemia medication was significantly higher in the controlled group than in the uncontrolled group.

A multivariate logistic regression analysis revealed the factors associated with therapeutic failure of hyperuricemia/ gout (Table 2). In Model 1, C2, C3, and C4 of the BMI were significantly positive associated with therapeutic failure compared to $\mathrm{C} 1(\mathrm{C} 2, \mathrm{AOR}=1.35,95 \% \mathrm{CI}=1.03-1.76 ; \mathrm{C} 3$, $\mathrm{AOR}=1.69,95 \% \mathrm{CI}=1.12-2.42 ; \mathrm{C} 4, \mathrm{AOR}=1.94,95 \% \mathrm{CI}=$ 1.26-2.98). In Model 2, C3 and C4 of the WC were significantly positive associated with therapeutic failure compared to $\mathrm{C} 1(\mathrm{C} 2, \mathrm{AOR}=1.29,95 \% \mathrm{CI}=0.95-1.75 ; \mathrm{C} 3, \mathrm{AOR}=1.41$, 95\% CI=1.01-1.97; C4, AOR=2.28, 95\% CI=1.63-3.18). In addition, taking dyslipidemia medication was significantly negative associated with therapeutic failure in both models (AOR=0.74 and 95\% $\mathrm{CI}=0.57-0.95$ in Model $1, \mathrm{AOR}=0.71$ and $95 \% \mathrm{CI}=0.55-0.92$ in Model 2). Those in the higher $\mathrm{BMI} / \mathrm{WC}$ categories were significantly more likely to have higher SUA levels than those in lower such categories (Fig. 2, 3).

\section{Discussion}

Our cross-sectional study revealed a low achievement rate of $37.5 \%$ for target SUA levels among Japanese men with hyperuricemia/gout. In addition, significant associations between therapeutic failure and BMI/WC categories were observed after adjusting for confounding factors. Increased 
Table 2. Factors Associated with Therapeutic Failure among Men (logistic Regression Analysis) $(\mathbf{n = 2 , 1 0 3 ) .}$

\begin{tabular}{|c|c|c|c|c|c|c|c|c|c|c|}
\hline & \multirow[b]{3}{*}{$\begin{array}{c}\text { Mean (SD) } \\
\text { or N (\%) }\end{array}$} & \multicolumn{3}{|c|}{ Univariate } & \multicolumn{6}{|c|}{ Multivariate } \\
\hline & & \multirow[b]{2}{*}{ OR } & \multirow[b]{2}{*}{$95 \% \mathrm{CI}$} & \multirow[b]{2}{*}{$\mathrm{p}$} & \multicolumn{3}{|c|}{ Model 1} & \multicolumn{3}{|c|}{ Model 2} \\
\hline & & & & & AOR & $95 \% \mathrm{CI}$ & $\mathrm{p}$ & AOR & $95 \% \mathrm{CI}$ & $\mathrm{p}$ \\
\hline \multicolumn{11}{|l|}{ Body mass index $\left(\mathrm{kg} / \mathrm{m}^{2}\right)$} \\
\hline $\mathrm{C} 1<25.0$ & $1,040(49.5)$ & & - & & & - & & & - & \\
\hline $25.0 \leq \mathrm{C} 2<27.5$ & $541(25.7)$ & 1.26 & $1.02-1.56$ & $*$ & 1.35 & $1.03-1.76$ & $* *$ & & - & \\
\hline $27.5 \leq \mathrm{C} 3<30.0$ & $299(10.6)$ & 1.49 & $1.14-1.96$ & $* *$ & 1.69 & $1.12-2.42$ & $*$ & & - & \\
\hline $30.0 \leq \mathrm{C} 4$ & $222(10.6)$ & 1.89 & $1.37-2.60$ & $* *$ & 1.94 & $1.26-2.98$ & $* *$ & & - & \\
\hline \multicolumn{11}{|l|}{ Waist circumference $(\mathrm{cm})$} \\
\hline $\mathrm{C} 1<85.0$ & $667(32.8)$ & & & & & & & & & \\
\hline $85.0 \leq \mathrm{C} 2<90.0$ & 487 (23.9) & 1.21 & $0.95-1.53$ & $*$ & & & & 1.29 & $0.95-1.75$ & \\
\hline $90.0 \leq \mathrm{C} 3<95.0$ & $374(18.4)$ & 1.24 & $0.96-1.61$ & $*$ & & & & 1.41 & $1.01-1.97$ & $*$ \\
\hline $95.0 \leq \mathrm{C} 4$ & $508(25.0)$ & 1.77 & $1.38-2.26$ & $* *$ & & & & 2.28 & $1.63-3.18$ & $* *$ \\
\hline Age (years) & $53.5(9.5)$ & 0.99 & $0.98-0.99$ & $*$ & 0.98 & $0.97-0.99$ & $* *$ & 0.97 & $0.96-0.99$ & $* *$ \\
\hline Hypertensive medication (yes) & $838(39.8)$ & 1.01 & $0.93-1.21$ & & 1.03 & $0.81-1.32$ & & 0.99 & $0.77-1.27$ & \\
\hline Diabetic medication (yes) & $128(6.1)$ & 0.96 & $0.66-1.39$ & & 0.92 & $0.59-1.43$ & & 0.90 & $0.57-1.41$ & \\
\hline Dyslipidemia medication (yes) & $545(25.9)$ & 0.81 & $0.66-0.99$ & $*$ & 0.74 & $0.57-0.95$ & $*$ & 0.71 & $0.55-0.92$ & $* *$ \\
\hline Alcohol consumption (non-daily drinker) & $1,195(56.8)$ & 1.04 & $0.87-1.24$ & & 0.80 & $0.64-1.01$ & & 0.81 & $0.64-1.04$ & \\
\hline Smoking behavior (non-smoker) & $1,468(69.8)$ & 1.02 & $0.82-1.27$ & & 0.99 & $0.76-1.31$ & & 1.07 & $0.81-1.41$ & \\
\hline eGFR (mL/min/1.73 m²) & $71.4(15.3)$ & 0.99 & $0.98-0.99$ & $* *$ & 0.98 & $0.97-0.99$ & $* *$ & 0.98 & $0.97-0.99$ & $* *$ \\
\hline
\end{tabular}

$\mathrm{N}$ : number, SD: standard deviation, OR: odds ratio, AOR: adjusted odds ratio, CI: confidence interval, eGFR: estimated glomerular filtration rate $* \mathrm{p}<0.05, * * \mathrm{p}<0.01$

Model 1 was adjusted for body mass index categories, age (years), hypertension medication (yes), diabetic medication (yes), dyslipidemia medication (yes), alcohol consumption (non-daily drinker), smoking (non-smoker), and eGFR ( $\mathrm{mL} / \mathrm{min} / 1.73 \mathrm{~m}^{2}$ ).

Model 2 was adjusted for waist circumference categories, age (years), hypertension medication (yes), diabetic medication (yes), dyslipidemia medication (yes), alcohol consumption (non-daily drinker), smoking (non-smoker), and eGFR ( $\left.\mathrm{mL} / \mathrm{min} / 1.73 \mathrm{~m}^{2}\right)$.

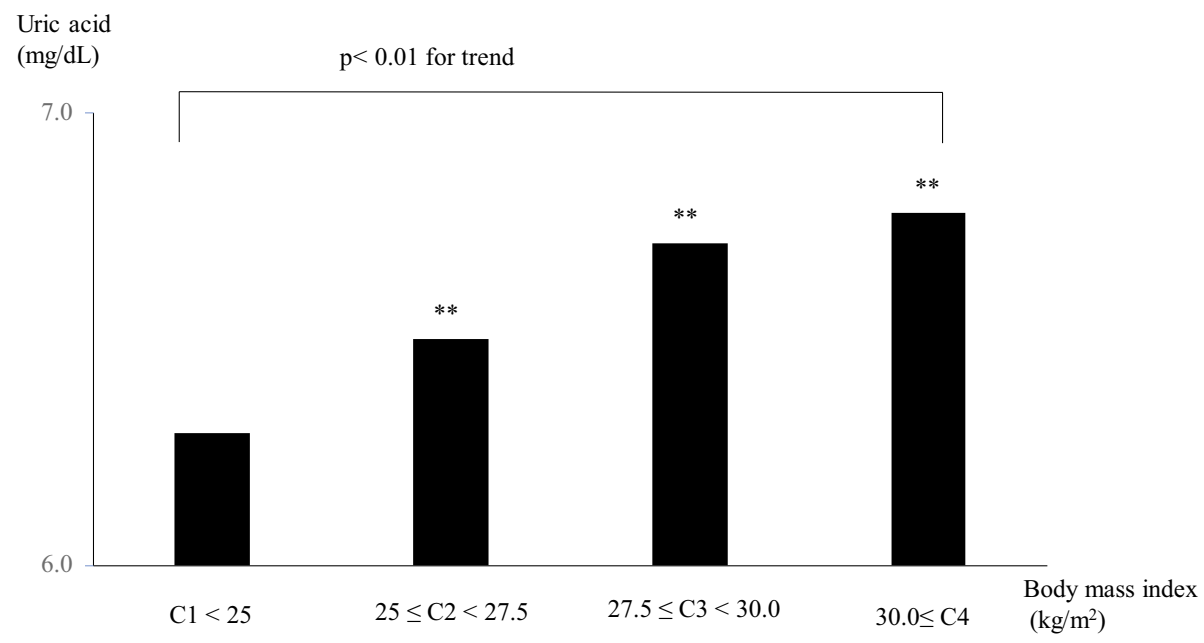

Figure 2. Relationship between the body mass index categories and the serum uric acid levels among men with hyperuricemia/gout.

BMI/WC categories showed a positive linear association with the SUA level. However, a medical history of dyslipidemia was a protective factor against therapeutic failure. To our knowledge, few studies have investigated the rates of success in treating hyperuricemia/gout or factors associated with therapeutic failures in the Japanese population; the present study therefore offers novel insight on this matter.

We observed low rates of achieving treatment goals among men with hyperuricemia/gout. Several experimental intervention studies have reported the efficacy of hyperuricemia medication $(17,18)$. One clinical trial, which was a randomized multi-center double-blind study conducted in Japan [mean age (SD): 51.4 (8.2) years], found that SUA levels $\leq$ $6.0 \mathrm{mg} / \mathrm{dL}(356.9 \mu \mathrm{mol} / \mathrm{L})$ were achieved in $76.9 \%$ of patients after they had been treated with $129 \mathrm{mg}$ topiroxostat (continuation rate: $89.0 \%$ ) (17). In another 12-week, multicenter, open-label, uncontrolled study in Japan [mean age (SD): 47.9 (11.1) years], the SUA levels at baseline and af- 


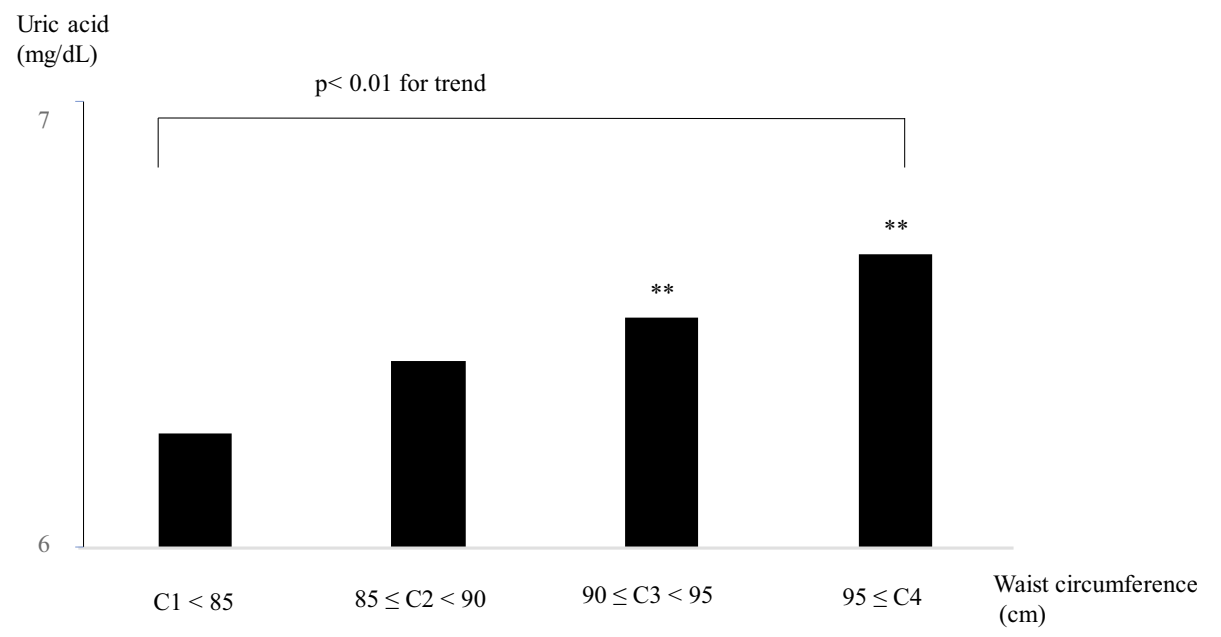

Figure 3. Relationship between the waist circumference categories and serum uric acid levels among men with hyperuricemia/gout.

ter administration of the antihyperuricemic fuxostat were reportedly $9.34 \pm 1.48$ and $5.59 \pm 1.17 \mathrm{mg} / \mathrm{dL}$, respectively, while those among normal excretors were 8.59 \pm 1.24 and $5.41 \pm 1.35 \mathrm{mg} / \mathrm{dL}$, respectively, and those among underexcretors were $8.29 \pm 1.01$ and $5.11 \pm 1.71 \mathrm{mg} / \mathrm{dL}$, respectively (18). Although these studies demonstrated high rates of achievement under several criteria, there are some discrepancies between their findings and ours. One possible explanation for this may be the awareness of target goals among physicians. A Japanese study conducted a questionnaire survey of 595 Japanese board-certified nephrologists for their criteria with regard to SUA levels in order to start urate-lowering therapy and identify a target level for chronic kidney disease with hyperuricemia (19). The survey reported respective start and target SUA levels of $8.2 \pm 0.9 \mathrm{mg} / \mathrm{dL}$ and $6.9 \pm 0.9 \mathrm{mg} / \mathrm{dL}, 8.4$ $\pm 0.9 \mathrm{mg} / \mathrm{dL}$ and $7.0 \pm 1.0 \mathrm{mg} / \mathrm{dL}, 8.6 \pm 1.0 \mathrm{mg} / \mathrm{dL}$ and $7.3 \pm 1.1$ $\mathrm{mg} / \mathrm{dL}$, and $9.1 \pm 1.2 \mathrm{mg} / \mathrm{dL}$ and $7.8 \pm 1.3 \mathrm{mg} / \mathrm{dL}$ at stages 3 , $4,4 \mathrm{D}$, and 5D, respectively, revealing a discrepancy between the target levels recommended by treatment guidelines and those recommended by physicians. Therefore, awareness among physicians must be improved in order to better manage SUA levels in line with treatment guidelines.

One other reason that may explain the discrepancy in our findings and others' is medication adherence among patients. A meta-analysis of data from 376,162 patients evaluated by 20 cardiovascular disease studies estimated an adherence rate of $57 \%$ across all studies (95\% CI, 50-64) after a median of 24 months (20). Although we did not assess the medication adherence within our study population, it may have been similar to that of previous studies and lower than that of experimental clinical trials. Thus, improved medication adherence may also be necessary in actual clinical settings in order to achieve better management of SUA levels.

Our study found that the BMI and WC were significantly associated with therapeutic failure among participants with hyperuricemia/gout. Previous reports have identified a positive association between obesity and uric acid levels (21-23). An epidemiological survey of 699 diabetic patients who had undergone an abdominal computed tomography assessment of the visceral fat area reported that a visceral fat area $>143$ $\mathrm{cm}^{2}$ was a significant independent predictor of hyperuricemia (OR 2.33, 95\% CI, 1.21-4.50, p=0.012), as opposed to a visceral fat area $<93 \mathrm{~cm}^{2}$, after adjusting for confounders (22). A large cross-sectional population study revealed in its SUA analysis that the chronic intake of sugarsweetened beverages was associated with an increased SUA level in the high-BMI group but not in the low-BMI group ( $p$ difference $=3.6 \times 10^{(-3)}$ ); the chronic intake of sugarsweetened beverages was also found to be associated with gout in the high-BMI group but not in the low-BMI group ( $p$ difference $=0.012$ ) (23). Several other interventional studies have revealed the impact of weight loss for improving hyperuricemia/gout $(24,25)$. The Multiple Risk Factor Intervention Trial, which analyzed 12,379 men with high cardiovascular risk profiles, reported that relative to those with no weight change (-0.9 to $0.9 \mathrm{~kg}$ ), the multivariate ORs of achieving normo-SUA levels after weight loss of 1-4.9 kg, $5-9.9 \mathrm{~kg}$, and $\geq 10 \mathrm{~kg}$ were 1.43 (95\% CI: $1.33,1.54), 2.17$ $(1.95,2.40)$, and $3.90(3.31,4.61)$, respectively, while the corresponding changes in SUA levels were $-0.12,-0.31$, and $-0.62 \mathrm{mg} / \mathrm{dL}(-7,-19$, and $-37 \mu \mathrm{mol} / \mathrm{L})(25)$. Thus, lifestyle modification to maintain an ideal body weight may be an option for treating hyperuricemia/gout.

Interestingly, our results indicate that having a medical history of taking dyslipidemia medication was a protective factor against therapeutic failure to meet SAU treatment goals. While this result seems contradictory, one potential explanation may be the association between SUA levels and lipid profiles $(26,27)$. A cohort study of 4,706 Chinese residents reported that per-unit increases in the BMI, BP, TG, and LDL-C levels were associated with 1.103-, 1.016-, $1.173-$, and 1.200-fold higher odds of the presence of hyperuricemia, and per-unit increases in HDL-C and FBG levels were associated with 0.616- and 0.900-fold lower odds of the presence of hyperuricemia, respectively (26). A retrospective 5-year cohort study of 6,476 healthy Japanese 
adults reported that a high baseline SUA was an independent risk factor for developing high LDL cholesterol in both men (OR: 1.159 per $1-\mathrm{mg} / \mathrm{dL}$ increase, 95\% CI:1.009-1.331) and women (OR: 1.215, 95\% CI: 1.061-1.390). Increased SUA levels for longer than 5 years also represented an independent risk factor for developing high LDL cholesterol levels and hypertriglyceridemia but not for developing low levels of HDL-C (27). In our study, the controlled group had lower LDL-C levels and higher HDL-C levels than the uncontrolled group. In addition, the LDL-C levels were significantly lower and the HDL-C levels higher in those taking dyslipidemia medication than in those who were not [LDLC: 112.1 (30.5) vs. 117.7 (29.2) mg/dL, HDL-C: 55.5 (14.1) vs. $52.7(11.8) \mathrm{mg} / \mathrm{dL}$ (data not shown)]. It is possible that better management by lowering lipid levels may help maintain SUA levels. Accordingly, comprehensive management, including that of lipid profiles, may be needed to ensure better treatment of hyperuricemia/gout.

Several limitations associated with the present study warrant mention. First, selection bias may have occurred, as participants comprised only those who underwent workplace medical checkups in Tokyo, Japan. As such, these participants may be inherently more aware of their health behaviors than residents in rural areas. Further analyses that include data from a more diverse cohort are needed. Second, some key data on items such as detailed information regarding medication, medication dosage, and medication adherence were not collected. Third, this study had a crosssectional design, so causal relationships between elevated SUA levels and obesity could not be evaluated. Further analyses of follow-up survey data will be needed to address this issue.

In conclusion, our large-scale cross-sectional study revealed low rates of achieving the target SUA level, and significant associations between therapeutic failure and the BMI/WC categories were observed after adjusting for confounders among participants with hyperuricemia/gout. We also found that the BMI/WC categories were positively associated with SUA levels, while a medical history of dyslipidemia was a protective factor against therapeutic failure. Collectively, these findings suggest a possible association between the rate of achieving target SUA levels and obesity while underscoring the importance of maintaining healthy lipid profiles as a way to manage uric acid levels. Better management of obesity in addition to that of dyslipidemia may help prevent the future development of cardiovascular disorders by maintaining healthy SUA levels.

The authors state that they have no Conflict of Interest (COI).

\section{Acknowledgement}

The authors thank all participants who underwent the voluntary workplace medical checkups as well as the data collection staff at the Tokyo Health Service Association.

Akiko Katayama and Hirohide Yokokawa contributed equally to this work

\section{References}

1. Frohlich ED. Uric acid. A risk factor for coronary heart disease. JAMA 270: 378-379, 1993.

2. Feig DI, Kang DH, Johnson RJ. Uric acid and cardiovascular risk. N Engl J Med 359: 1811-1821, 2008.

3. Wang S, Shu Z, Tao Q, Yu C, Zhan S, Li L. Uric acid and incident chronic kidney disease in a large health check-up population in Taiwan. Nephrology 16: 767-776, 2011.

4. Nagahama K, Inoue T, Kohagura K, Ishihara A, Kinjo K, Ohya Y. Hyperuricemia predicts future metabolic syndrome: a 4-year follow-up study of a large screened cohort in Okinawa, Japan. Hypertens Res 37: 232-238, 2014.

5. Mazzali M, Hughes J, Kim YG, et al. Elevated uric acid increases blood pressure in the rat by a novel crystal-independent mechanism. Hypertension 38: 1101-1106, 2001.

6. Kang DH, Nakagawa T, Feng L, et al. A role for uric acid in the progression of renal disease. J Am Soc Nephrol 13: 2888-2897, 2002.

7. Nakagawa T, Hu H, Zharikov S, et al. A causal role for uric acid in fructose-induced metabolic syndrome. Am J Physiol Renal Physiol 290: 625-631, 2006.

8. Lim SY, Lu N, Oza A, et al. Trends in gout and rheumatoid arthritis hospitalizations in the United States, 1993-2011. JAMA 315: 2345-2347, 2016.

9. Japanese Society of Gout and Nucleic Acid Metabolism. Japanese guideline for the management of hyperuricemia and gout: 2nd ed. 2012 [Internet]. [cited 2018 Jun. 15]. Available from: http://www.t ukaku.jp/wp-content/uploads/2013/06/tufu-GL2.pdf (in Japanese).

10. Shoji A, Yamanaka H, Kamatani N. A retrospective study of the relationship between serum urate level and recurrent attacks of gouty arthritis: evidence for reduction of recurrent gouty arthritis with antihyperuricemic therapy. Arthritis Rheum 51: 321-325, 2004.

11. Iseki K, Oshiro S, Tozawa M, Ikemiya Y, Fukiyama K, Takishita $\mathrm{S}$. Prevalence and correlates of diabetes mellitus in a screened cohort in Okinawa, Japan. Hypertens Res 25: 185-190, 2002.

12. Shiozawa A, Szabo SM, Bolzani A, Cheung A, Choi HK. Serum uric acid and the risk of incident and recurrent gout: a systematic review. J Rheumatol 44: 388-396, 2017.

13. Warnick GR, Knopp RH, Fitzpatrick V, Branson L. Estimating low-density lipoprotein cholesterol by the Friedewald equation is adequate for classifying patients on the basis of nationally recommended cut points. Clin Chem 36: 15-19, 1990.

14. Kashiwagi A, Kasuga M, Araki E, et al. Committee on the Standardization of Diabetes Mellitus-Related Laboratory Testing of Japan Diabetes Society. International clinical harmonization of glycated hemoglobin in Japan: from Japan Diabetes Society to National Glycohemoglobin Standardization Program values. J Diabetes Investig 3: 39-40, 2012.

15. Belloc NB, Breslow L. Relationship of physical health status and health practices. Prev Med 1: 409-421, 1972.

16. Yokokawa H, Goto A, Sanada H, et al. Achievement status toward goal blood pressure levels and healthy lifestyles among Japanese hypertensive patients; cross sectional survey results from Fukushima Research of Hypertension (FRESH). Intern Med 50: 1149-1156, 2011.

17. Hosoya T, Sasaki T, Ohashi T. Clinical efficacy and safety of topiroxostat in Japanese hyperuricemic patients with or without gout: a randomized, double-blinded, controlled phase $2 \mathrm{~b}$ study. Clin Rheumatol 36: 649-656, 2017.

18. Yamamoto T, Hidaka $Y$, Inaba $M$, et al. Effects of febuxostat on serum urate level in Japanese hyperuricemia patients. Mod Rheumatol 25: 779-783, 2015. 
19. Nakaya I, Namikoshi T, Tsuruta Y, et al. Management of asymptomatic hyperuricaemia in patients with chronic kidney disease by Japanese nephrologists: a questionnaire survey. Nephrology 16: 518-521, 2011.

20. Naderi SH, Bestwick JP, Wald DS. Adherence to drugs that prevent cardiovascular disease: meta-analysis on 376,162 patients. Am J Med 125: 882-887, 2012.

21. Nakanishi N, Yoshida H, Nakamura K, Suzuki K, Tatara K. Predictors for development of hyperuricemia: an 8-year longitudinal study in middle-aged Japanese men. Metabolism 50: 621-626, 2001.

22. Kim TH, Lee SS, Yoo JH, et al. The relationship between the regional abdominal adipose tissue distribution and the serum uric acid levels in people with type 2 diabetes mellitus. Diabetol Metab Syndr 4: 3, 2012.

23. Dalbeth N, Phipps-Green A, House ME, et al. Body mass index modulates the relationship of sugar-sweetened beverage intake with serum urate concentrations and gout. Arthritis Res Ther 17: 263, 2015.

24. Nielsen SM, Bartels EM, Henriksen M, et al. Weight loss for overweight and obese individuals with gout: a systematic review of longitudinal studies. Ann Rheum Dis 76: 1870-1882, 2017.

25. Zhu Y, Zhang Y, Choi HK. The serum urate-lowering impact of weight loss among men with a high cardiovascular risk profile: the Multiple Risk Factor Intervention Trial. Rheumatology 49: 23912399, 2010.

26. Fu S, Luo L, Ye P, Xiao W. Epidemiological associations between hyperuricemia and cardiometabolic risk factors: a comprehensive study from Chinese community. BMC Cardiovasc Disord 15: 129, 2015.

27. Kuwabara M, Borghi C, Cicero AFG, et al. Elevated serum uric acid increases risks for developing high LDL cholesterol and hypertriglyceridemia: a five-year cohort study in Japan. Int J Cardiol 261: 183-188, 2018.

The Internal Medicine is an Open Access journal distributed under the Creative Commons Attribution-NonCommercial-NoDerivatives 4.0 International License. To view the details of this license, please visit (https://creativecommons.org/licenses/ by-nc-nd/4.0/).

(C) 2019 The Japanese Society of Internal Medicine

Intern Med 58: 1225-1231, 2019 